\title{
Unacceptable Results with an Accepted Soft Tissue Filler: Polyacrylamide Hydrogel
}

\author{
Z. Paul Lorenc
}

Received: 5 May 2010/ Accepted: 5 May 2010/Published online: 7 July 2010

(c) Springer Science+Business Media, LLC and International Society of Aesthetic Plastic Surgery 2010

All injectable volumizing agents and fillers may be associated with immediate or delayed complications (overall rate, $<1 \%$ ) [1]. The complication rate is directly related to the technique of injection as well as to the properties of the injected material. With increased persistence of the material, the possibilities of serious complications become more dependent on the inherent physiochemical characteristics of the material rather then on those of the injection technique.

Polyacrylamide is used for protein separation in molecular biology [2]. The clinical use of polyacrylamide gel (PAG) as an injectable filler started as Interfall in the Ukraine in 1983. In small quantities, the gel is well tolerated and slowly absorbed by the body (t1/2, 20 years). Different preparations of polyacrylamide hydrogels have unique characteristics, although they all are a combination of a hydrophilic polyacrylamide and nonpyrogenic water.

The PAGs discussed in the article by Manafi and colleagues have very different characteristics in terms of the gel concentration. Interfall (Interfall Ltd., Kiev, UA) is a hydrogel with a variable gel concentration of $3.5 \%$ to $9 \%$. The latest PAG that has undergone PMA submission to the Food and Drug Administration (FDA) is Aquamid hydrogel [3], a consistent $2.5 \%$ cross-linked polyacrylamide gel manufactured by Contura (Denmark). Aquamid (Contura International, Switzerland) is a gel with a constant PAG concentration of $5 \%$. When the safety profile and efficacy of an injectable material is evaluated, all of its unique characteristics must be discussed so an appropriate conclusion can be drawn.

Z. P. Lorenc $(\bowtie)$

Aesthetic Plastic Surgery, 983 Park Avenue, New York, NY 10028, USA

e-mail: lorenc@lorenc.com
In the article by Manafi and colleagues, the patient basis was biased in the conclusion drawn. Only patients experiencing unsatisfactory results and complications of polyacrylamide hydrogel injections were included in their study. Three different preparations of PAG materials were included, yet the effect of the gel concentration on the complication rate was not discussed. An in-depth discussion on the relationship of the volume injected $(200 \mathrm{ml} /$ breast for case 2 vs. $5 \mathrm{ml} /$ lips for case 1) would have been welcome because our experience and clinical data in relation to permanent and semipermanent fillers supported it [1].

Although this article is of clinical interest, the conclusion drawn is not based on the data presented. Recent data [3, 4] from multicenter double-blind studies of 315 and 101 patients, respectively, have shown injections of polyacrylamide hydrogel for soft tissue augmentation at 12 and 24 months to be safe, efficacious, and without adverse soft tissue reactions. In the United States, PMA has submitted Aquamid hydrogel to the FDA.

\section{References}

1. Lemperle $G$ et al (2006) Avoiding and treating dermal filler complications. PRS 118:3S, 92

2. Lemperely G et al (2003) Human histology and persistence of various injectable filler substances. Aesth Plast Surg 27:354

3. Rohrich R et al (2009) A 12-month controlled study in the US of the safety and efficacy of Aquamid hydrogel. PRS 124:4S, 12

4. Buelow S (2006) Efficacy and safety of polyacrylamide hydrogel for facial soft-tissue augmentation in a 2-year follow-up. PRS 118:3S, 85 